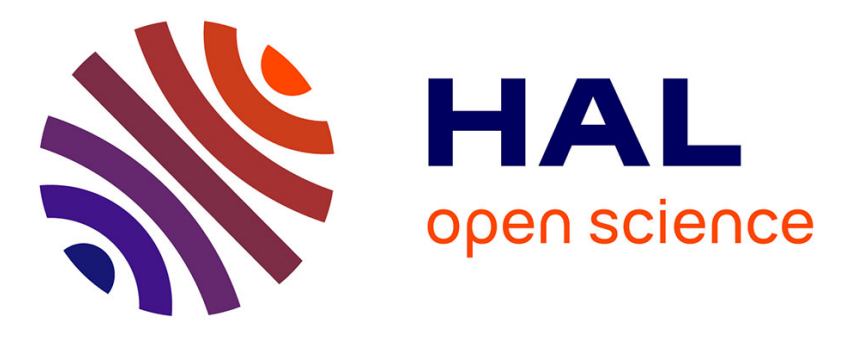

\title{
Investigating the semantic perceptual space of synthetic textures on an ultrasonic based haptic tablet
}

Maxime Dariosecq, Patricia Plénacoste, Florent Berthaut, Anis Kaci, Frédéric Giraud

\section{- To cite this version: \\ Maxime Dariosecq, Patricia Plénacoste, Florent Berthaut, Anis Kaci, Frédéric Giraud. Investigating the semantic perceptual space of synthetic textures on an ultrasonic based haptic tablet. HUCAPP 2020, Feb 2020, Valletta, Malta. hal-02434298}

\section{HAL Id: hal-02434298 \\ https://hal.science/hal-02434298}

Submitted on 9 Jan 2020

HAL is a multi-disciplinary open access archive for the deposit and dissemination of scientific research documents, whether they are published or not. The documents may come from teaching and research institutions in France or abroad, or from public or private research centers.
L'archive ouverte pluridisciplinaire HAL, est destinée au dépôt et à la diffusion de documents scientifiques de niveau recherche, publiés ou non, émanant des établissements d'enseignement et de recherche français ou étrangers, des laboratoires publics ou privés. 


\title{
Investigating the semantic perceptual space of synthetic textures on an ultrasonic based haptic tablet
}

\author{
Maxime Dariosecq ${ }^{1}$, Patricia Plénacoste ${ }^{1}$, Florent Berthaut ${ }^{1}$, Anis Kaci ${ }^{2}$ and Frédéric Giraud ${ }^{2}$ \\ ${ }^{1}$ CRIStAL, CNRS, University of Lille, France \\ ${ }^{2}$ L2EP, University of Lille, France \\ firstname.lastname@univ-lille.fr
}

\begin{abstract}
Keywords: Haptic feedback; Tactile device; Ultrasonic vibrations; Perceptual space; Material perception; Texture rendering

Abstract: $\quad$ This paper aims to investigate the semantic perceptual space of synthetic tactile textures rendered via an ultrasonic based haptic tablet and the parameters influencing this space. Through a closed card sorting task, 30 participants had to explore 32 tactile-only textures and describe each texture using adjectives. A factorial analysis of mixed data was conducted. Results suggest a 2 dimensional space with tactile textures belonging to a continuum of rough to smooth adjectives. Influence of waveform and amplitude is shown to play an important role in perceiving a texture as smooth or rough, and spatial period is a possible modulator of different degrees of roughness or smoothness. Finally, we discuss how these findings can be used by designers on tactile feedback devices.
\end{abstract}

\section{Introduction}

The vast majority of our daily touch interactions on devices such as phones or tablets, consisting mainly of visual and auditory information (Levesque et al., 2011), are still lacking colourful tactile experience, which has been shown to add to their emotional impact (Wilson and Brewster, 2017). While real materials vary in shape but also size or textures, the simulation of these materials is often limited, due to the technological constraints of the devices. As such, various devices can be found, ranging from deformation crust devices, pin arrays, electrovibratory devices, midair vibrations or friction modulation surfaces (Culbertson et al., 2018). Focusing specifically on tactile textures, ultrasonic based tablets have shown promising enhancements to the user's exploratory movements, allowing applications such as haptic text messages, image sharing and virtual touch (Mullenbach et al., 2014) or haptic e-books (Cingel and Piper, 2017) which can enhance children's reading activity on a tablet with tactile textures. These devices rely on a phenomenon called squeeze film effect (Biet et al., 2007) which leverages ultrasonic vibrations to create an air-gap between the user's finger and the tablet, resulting in friction reduction.

\subsection{Related Work}

Thus far, most of the studies on tactile perception conducted on these devices were focused on perception thresholds of friction, size or sharpness(Biet et al., 2007; Samur et al., 2009; Kalantari et al., 2016; Kalantari et al., 2018b; Kalantari et al., 2018a). In the context of interaction design however, many opportunities will arise from the ability of rendering tactile textures that provide users with specific perceptual sensations (i.e. rough/smooth, soft/hard). Little research has addressed the perception of simulated textures (Brewer et al., 2016; Messaoud et al., 2016). In these studies, real and simulated surfaces were explored and compared by the participants. Results show acceptable classifications between real and re-created textures, therefore illustrating the influence of parameters such as temporal frequency and spatial period, amplitude, friction, with specific signal shapes. Fabrics or material with unique extracted features gave participants enough perceptual cues to successfully distinguish between compliant and harder surfaces, between low friction surfaces and high friction surfaces, but also between different recreated fabric such as velvet and cotton.

In Messaoud (Messaoud et al., 2016) virtual tactile textures were created using a multi level approach with both signal shape (the average of user's friction 
contrast) and real textile specific spatial periods (numbers of grooves of the textile's surface) gathered by tribological extraction. Instead of relying on existing materials, we focus here on synthetic textures, with the goal of allowing interface designers to generate semantically defined (e.g. smooth, rough ...) textures from a set of parameters. A similar approach was proposed by Friesen et al (Friesen et al., 2018), who study the users' ability to recognise the spatial frequency of synthetic tactile textures. We believe that observing how individuals perceive and give perceptual descriptors to randomly simulated textures will offer an insight into which parameters tend to influence perception. In this paper, we aim to strengthen this methodology by giving a more general view about the influence of these parameters on the user's perception, allowing designers to approach specific tactile perceptions without the need of tribological extractions of real materials.

Human perceptual and affective experiences in touching materials are expressed by a semantically multilayered and multidimensional information space (Okamoto and Ho., 2016). The bottom layer of this perception system involves the perception of physical properties, allowed by tactile receptors distributed by clusters around the human skin. Activated mechanoreceptors or thermoreceptors will then transmit information through nerve signals to the brain (Dargahi and Najarian, 2004). This psychophysical layer, or layer of textures has been widely investigated, with more than 20 papers offering a relatively homogeneous description of the dimensions required to identify textures. While results may vary, previous attempts to describe this texture perception space used multidimensional scaling (MDS) to converge to an optimal 3 to 5 dimensions space (Holliins et al., 1993; Hollins et al., 2000; Picard et al., 2003; Tiest and Kappers, 2006; Guest et al., 2011; Okamoto and Ho., 2016). These main textural dimensions supposedly include continuum such as "Rough/Smoothness", "Hard/softness", "Sticky/Slipperiness", "Hot/cold" and "Dry/Wetness". Going further, Guest et al. (Guest et al., 2011) broadened the lexicon used to describe the experience of touch using similar MDS based experimental design to represent the semantic perceptual spaces underlying the words answered in touch perception tasks. If the importance of a clear lexicon to describe the perceptual experiences of real materials is stressed by Guest et al., a lexicon describing artificially rendered textures on haptic devices would greatly help further developing these technologies.

The semantic perception space for real materials has been thoroughly studied, but it remains mostly unknown for synthetic tactile textures on an ultrasonic tablet. Moreover, there are no clear guidelines on how to tune the parameters used for texture rendering to elicit a specific perception. The goal of this study is to broadly investigate the user's semantic perceptual space of ultrasonic rendered textures. We also aim to reveal the relationship between specific semantic patterns and the synthesis parameters used to create the textures. More precisely, we aim to observe if there is a specific relation between the waveform, amplitude, and frequency and the descriptors used to qualify the perception of specific textures.

\section{Experiment}

A closed card sorting task was performed to investigate the participant's semantic perceptual space after they explored a sample of synthetic tactile texture. Participants had to explore simulated textures on an ultrasonic tactile device and qualify each texture using descriptors of either consistency and/or texture. It is worth noting that there was no visual feedback for the participants, the screen remained blank and the textures were only tactile. Textures were explored only once, without time restriction and with the preferred hand. During this task noise-cancelling headphones were used to prevent interference with noises that could be emitted by the device. Factor analysis of mixed data was used to investigate both the semantic perceptual space derived from the chosen adjectives and its relation with the texture's parameters.

\subsection{Textures and descriptors}

Thirty two synthetic tactile textures were generated on the tablet's surface using different waveforms, spatial periods, amplitudes and ratios with a constant offset. Two waveforms were used, namely sine and square waves which is depicted in Figure 1. Spatial periods ranged from 1000 to $10000 \mu \mathrm{m}$ including 1000, 2500, 5000 and $10000 \mu \mathrm{m}$. Ratios, which correspond to the proportion of high voltage in a period for the square waveform, consisted of 10, 50, and 90 percents. Finally, two amplitudes ranges of $10 \%$ and $40 \%$ were used with an offset of $50 \%, 100 \%$ being the maximal voltage actuated by the piezoelectric cells.

Participants were asked to describe textures by selecting adjectives from a set of 20 descriptors. Instead of relying on descriptors designed for visual textures (Rao and Lohse, 1996), we chose to build a tactile specific set of descriptors. They were therefore chosen by narrowing down answers from a pre-testing open card sorting task with 22 participants in which 

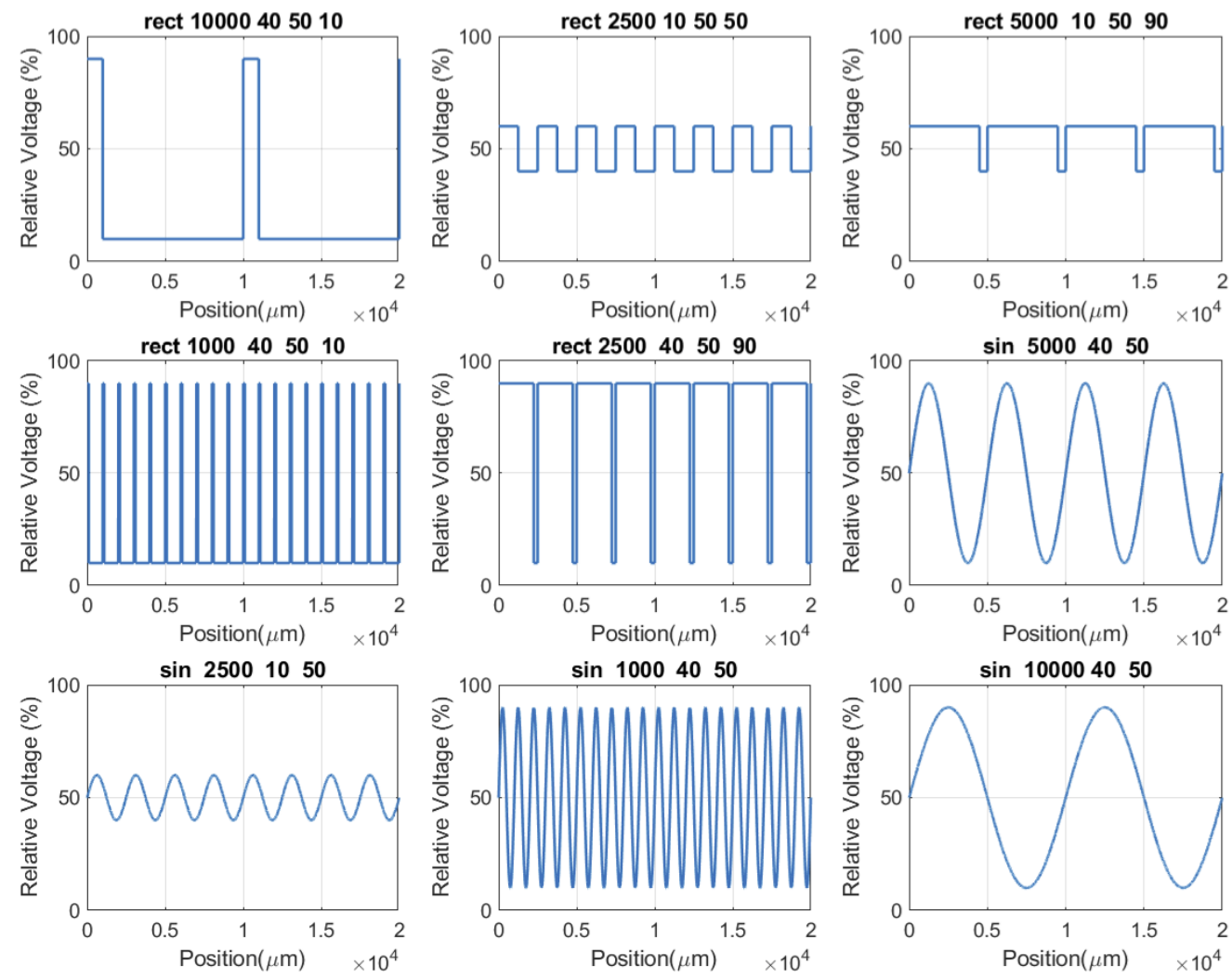

Figure 1: Example textures from a selection of waveforms (sine and rect/square waves), spatial periods, amplitude and ratios (only for square waves) used in the study. 50 corresponds to the offset (50\% of voltage)

they were asked to propose as many adjectives as they wanted on the same set of synthetic textures. From these answers, the ten more frequent adjectives were kept. This pre-testing task also revealed a number of terms describing consistency rather than texture, which we chose to keep as a separate category and complete with additional adjectives.

\begin{tabular}{|c|c|}
\hline Texture & Consistency \\
\hline Delicate & Hard \\
Spiky & Soft \\
Sticky & Mellow \\
Rough & Pasty \\
Smooth & Slimy \\
Granular & Elastic \\
Wet & Spongy \\
Oily & Gelatinous \\
Sandy & Viscous \\
Slippery & Friable \\
\hline
\end{tabular}

Table 1: Summary of the descriptors

\subsection{Apparatus}

Friction reduction comes with a number of limitations, such as the absence of feedback when the finger is not moving. However it allows for finer control of synthetic texture parameters compared to other technologies such as vibrotactile feedback, which would not permit the range of spatial periods that we investigate in this study.

A tactile feedback display based on ultrasonic vibrations for haptic rendering was used, which is depicted in Figure 2. The enhanced visual tactile actuator (E-ViTa) is a Banana Pi based single-board computer (Shenzhen LeMaker Technology Co. Ltd, China),running a $1 \mathrm{GHz}$ ARM Cortex-A7, a dual-core CPU and 1 GB RAM. A STM32f4 microcontroller (STMicroelectronics, France) is allowed to work in parallel with the computer via Serial Peripheral Interface (SPI) bus at $10 \mathrm{kHz}$. To be able to detect positions of the fingers, this setup is connected to a $12.5 \mathrm{~cm}$ capacitive touchscreen (Banana-LCD 5"-TS, MAREL, China), with a sampling frequency of 62 
$\mathrm{Hz}$.

Ten $1460.5 \mathrm{~mm}$ piezoelectric cells actuate a $154811.6 \mathrm{~mm}$ fixed glass plate, resonating at 60750 $\mathrm{Hz}$ with a half wavelength of $8 \mathrm{~mm}$. A power electronic circuit converts a $12 \mathrm{~V}$ DC voltage source into an AC voltage, controlled in amplitude and frequency and supplied to the piezoelectric cells. The microcontroller synthesizes a pulse-width modulation (PWM) signal to drive a voltage inverter that actuates the piezoceramics. Details on the friction reduction which can be achieved with this technology can be found in (Vezzoli et al., 2017).

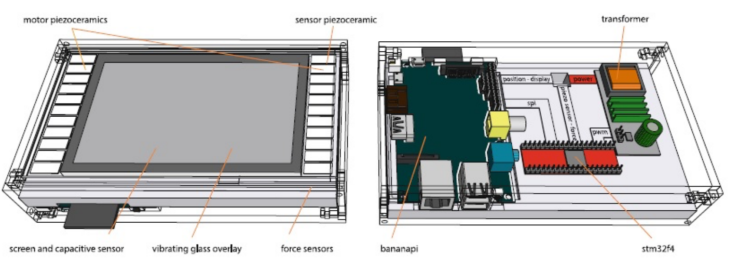

Figure 2: Structure of the E-Vita ultrasonic based haptic display

\subsection{Participants}

A sample of 30 participants (24 males, 6 females, $\mathrm{M}=29, \mathrm{SD}=8.8$ ) were recruited for this experiment. They were all naive regarding the haptic technology used in the experiment to avoid any interference during the response phase of the protocol. They where all right-handed, without motor impairment, and participated freely in the experiment.

\subsection{Procedure}

Participants were seated and briefed in front of the setup (see Figure 3). As proposed by Rao et al. (Rao and Lohse, 1996) for visual textures, the 32 synthetic tactile textures were presented one at a time and only once, in random order. The textures were only tactile and presented on a blank screen, i.e. the textures did not have a visual representation presented to the participants. Participant were free to explore each texture for as long as they needed, using a continuous motion with their index finger. The hardware used for this experiment does not allow for multitouch tactile feedback, so we restrained the exploration to a single finger. They then had to choose between the 20 descriptors listed in Table 1 on a laptop placed next to the tablet. Participants were instructed to choose descriptors they deemed accurate to represent the explored texture. There was no limitation regarding the number of descriptors used. Experiment duration averaged 35 minutes.

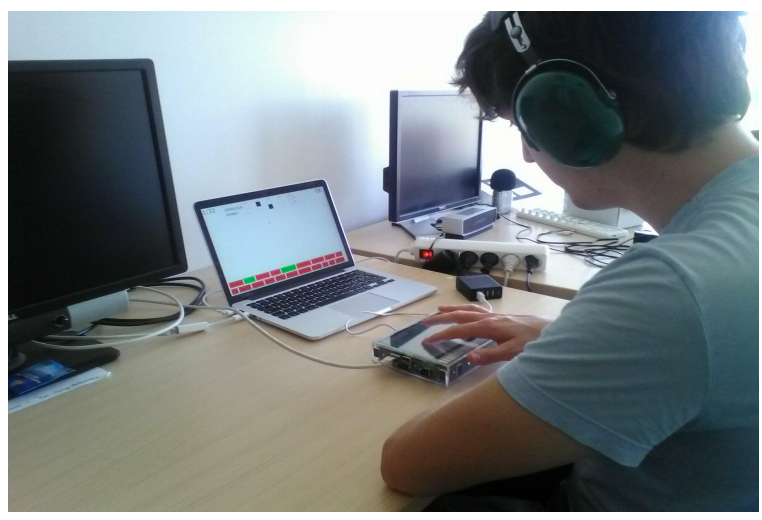

Figure 3: Experimental setup

\subsection{Statistical analysis}

A $2 \mathrm{~d}$ square matrix was computed using the participants' answers to each texture. Each cell expresses the probability of each adjective being chosen for a given texture.

As pointed out by Okamoto et al. (Okamoto and Ho., 2016), factor analysis is a commonly used strategy when assessing the psychophysical dimensions of tactile perception of textures. However introducing both qualitative (the texture's parameters) and quantitative (the probability of an adjective occurring) variables requires the use of a factor analysis of mixed data (FAMD). For each texture, we have $\mathrm{K}=4$ quantitative variables (the waveform, spatial period, amplitude, ratio parameters) on a lower two dimensional space, and $\mathrm{Q}=20$ quantitative variables (probability for each adjective) on a related subspace while maximizing inertia. Thus, after testing for correlation of both $\mathrm{K}$ and $\mathrm{Q}$ dimensions, independent factors are constructed representing a maximum portion of variance from the original data. Each factor can be considered as a perceptual dimension (Okamoto and Ho., 2016).

\subsection{Excluded textures and descriptors}

After running this model a first time, a selection of textures and descriptors were removed from the model, because they showed either a very low probability of occurrence, no correlations, or absence of clear patterns. The following results will not account for the following descriptors : hard, mellow, pasty and spongy and the textures presented in table 2 . 

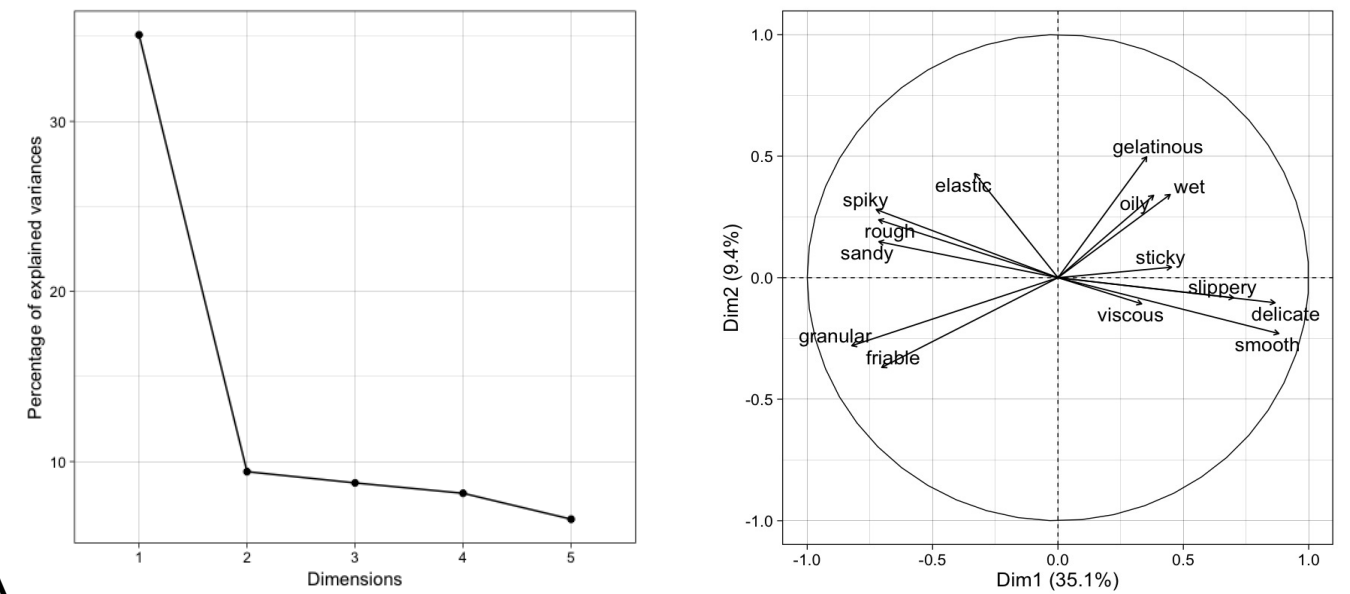

B
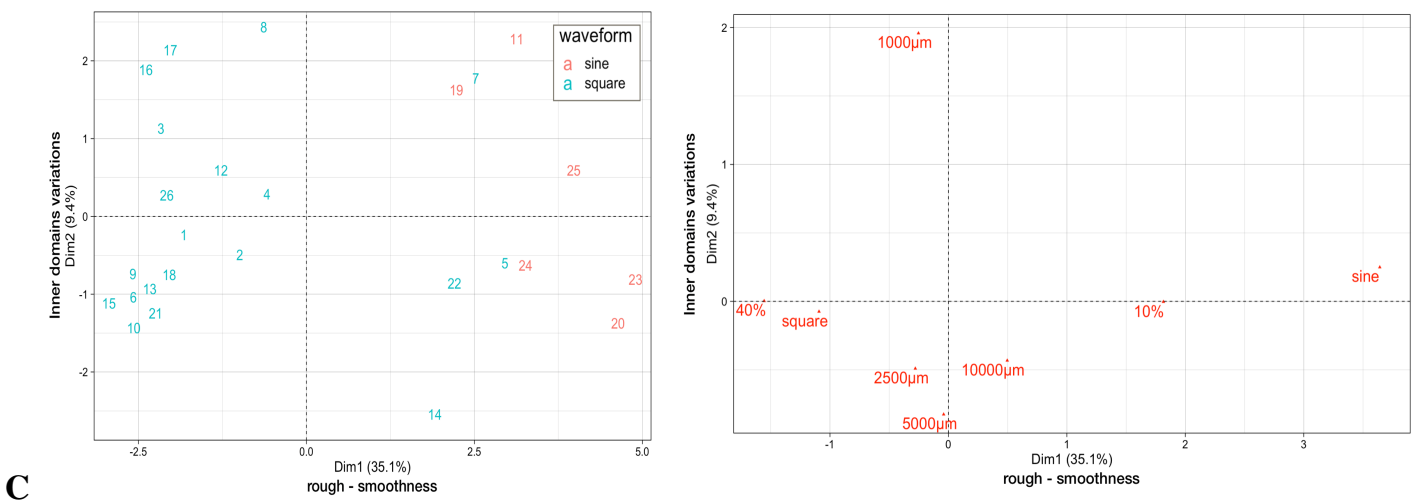

Figure 4: FAMD plots. A) screeplot, B) correlation circle for quantitative variables, C) subspace projection for the textures, D) subspace projection for the quantitative variables (waveform : $\sin (1) /$ square(2), amplitude : $10 \% / 40 \%$, period : $1000 / 2500 / 5000 / 10000(\mu \mathrm{m})$

\begin{tabular}{|lllll|}
\hline \multicolumn{2}{|c|}{ Texture } & Waveform Period & \multicolumn{2}{c|}{ Amplitude Ratio } \\
\hline 1 & rect & 1000 & 10 & 90 \\
16 & rect & 10000 & 10 & 50 \\
19 & rect & 5000 & 10 & 50 \\
23 & rect & 2500 & 10 & 50 \\
27 & sin & 2500 & 40 & na \\
28 & sin & 1000 & 40 & na \\
\hline
\end{tabular}

Table 2: Removed textures

\section{Results and discussion}

\subsection{Semantic perceptual space}

Results for the factor analysis of mixed data demonstrate an optimal two dimensional space as shown by the scree plot in Figure 4.A with a total of 44.6 $\%$ variance retained. Factor $1(35.1 \%)$ can be interpreted as a rough/smoothness continuum with semantically opposed clusters of descriptors including rough descriptors all correlating negatively with F1 and smooth descriptors positively correlating (Figure 4.B). Table 3 shows the loading values for all factors, all loadings appearing as their maximum values. Loadings values can be interpreted as the correlation coefficient between the loading and the factor. All descriptors within F1 show correlation coefficients superior to .71 , indicating a strong relationship to the factor. With approximately 3 times the variance retained, the rough/smoothness factor accounts for most of the variations in this model, although F2's (9.4\%) loadings tends to explains both smooth and rough inner clusters variations, with either rougher or smoother surfaces. These results indicate that a methodology designed to assess the psychophysical dimensions (Okamoto and Ho., 2016) and the semantic (Guest et al., 2011) of tactile perception on real textures can be transferred to rendered ultrasonic textures. The texture sample used for this study therefore seem to elicit perception of various roughness levels. Perception elements of viscoelasticy (F3, 8.74\%) and 


\begin{tabular}{|c|c|c|c|c|}
\hline Factor & $\begin{array}{l}\text { F1: rough / } \\
\text { smoothness }\end{array}$ & F2: inner domains variations & F3 : viscoelasticity & F4: wet/stickyness \\
\hline \%variance & $35 \%$ & $9.4 \%$ & $8.74 \%$ & $8.13 \%$ \\
\hline $\begin{array}{l}\text { Relationship } \\
\text { squared } \\
\text { (qualitative) }\end{array}$ & $\begin{array}{l}\text { Waveform } .60 \\
\text { amplitude } .42\end{array}$ & period .63 & & \\
\hline $\begin{array}{l}\text { Loadings } \\
\text { (quantitative) }\end{array}$ & $\begin{array}{l}\text { granular }-.82 \\
\text { spiky }-.72 \\
\text { rough }-.72 \\
\text { sandy }-.71 \\
\text { friable }-.71 \\
\text { smooth } .88 \\
\text { delicate } .86 \\
\text { slippery } .70\end{array}$ & & $\begin{array}{l}\text { viscous } .52 \\
\text { elastic } .45\end{array}$ & $\begin{array}{l}\text { sticky }-.60 \\
\text { wet } .50\end{array}$ \\
\hline
\end{tabular}

Table 3: FAMD factors extracted from the probability of adjective occurrence given a texture.

wet/stickyness $(\mathrm{F} 4,8.13 \%)$ can also be extracted.

\subsection{Relation between textures parameters and semantic perceptual space}

In order to elicit specific perceptions, the underlying influence of the parameters used to render these textures must be addressed. By maximizing the correlations between qualitative and quantitative elements and the factors, factor analysis of mixed data offers a tool to assess these relations. Figure 4.C represents the texture's sub-space within the factors. We chose not to provide the details for each of the 32 individual textures, to focus on the overall texture parameters. Figure 4.D shows each modality of the texture's parameters, appearing as the centroid of the textures possessing these properties in the $2 \mathrm{D}$ factor space. Results show relationship squared coefficients of .60 for waveform and .42 for amplitude with F1, and .63 between period and F2. Relationship squared metric helps appreciate the strength of the relation between a qualitative variable and the factor.

Figure 4.D and Table 3 show a strong influence of waveform and amplitude on F1, meaning that these variables influence heavily the participant's perception, leading them to categorize a texture on a rough or smooth continuum. Indeed, textures being identified as rough-like (Figure 4.C), tend to possess specific properties such as being square waveforms with higher amplitude, while smoother textures tend to be sinusoidal waveforms with lower amplitudes. Variations within either continuum could here be explained by the period, indeed variations located on the vertical axis (Figure 4.B-dim2, Figure 4.D-dim2) of dif- ferent roughness or softness tend to vary with different magnitudes of periods. Also, Figure 4.C shows a small cluster of square waveforms being identified as smooth-like textures, due to lower amplitudes and a probable effect of ratio, participants might not be able to clearly perceive elements of macro roughness with such reliefs.

\subsection{Limitations}

Regarding limitations, this experiment does not provide a specific list of textures eliciting very precise and consistent perceptions. It rather provides general guidelines allowing one to play with the dimension of rough/smooth textures. As pointed out by Okamoto et al. (Okamoto et al., 2013), adjective labels used in these experiments are suffering from limitations, such as limited vocabulary related to physical properties of material, semantic variability or the lack of consensus regarding the meaning of a descriptor. More appropriately, these descriptors are more often used for the detection of perceptual dimensionality, it is no surprises that the descriptors presenting the best correlations, probability of occurrences and representation in this model tend to belong to the description of perceptual dimensions.

\subsection{Implications for the design of tactile feedback}

From the results, we can extract a set of texture parameters that elicit perceptions ranging from smooth to rough. This set is shown in Table 4. Figure 5 depicts the corresponding waveform patterns used for texture rendering. As shown by our results, elicit- 


\begin{tabular}{|c|c|c|c|c|}
\hline \multicolumn{5}{|l|}{ Adjective } \\
\hline granulous & rect & 5000 & 40 & 50 \\
\hline rough & rect & 2500 & 40 & 50 \\
\hline sandy & rect & 1000 & 40 & 50 \\
\hline smooth & $\sin$ & 10000 & 10 & na \\
\hline delicate & $\sin$ & 1000 & 10 & na \\
\hline
\end{tabular}

Table 4: Tactile perception adjectives and possible corresponding synthetic texture parameters
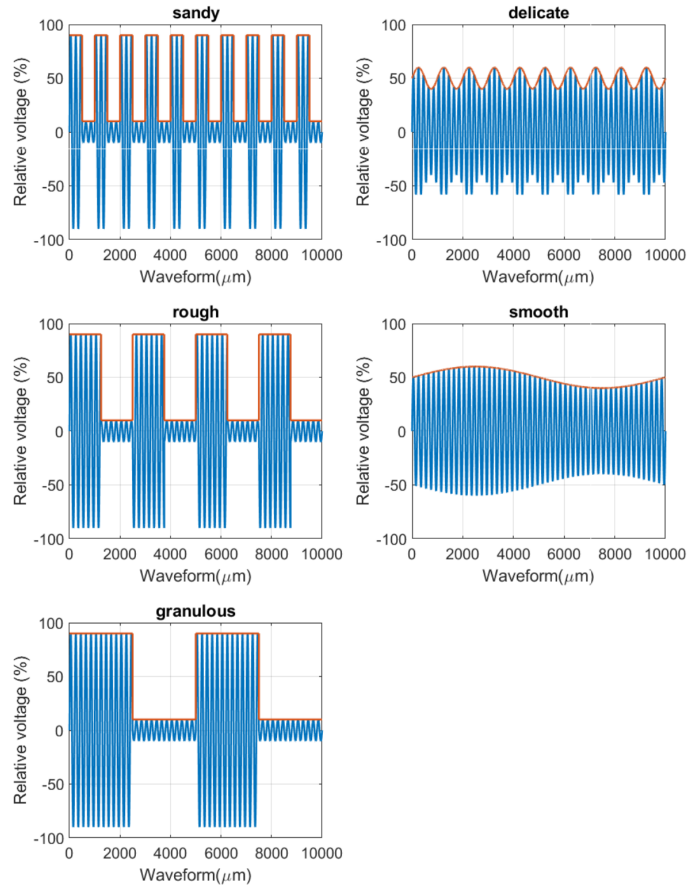

Figure 5: Waveform sample resulting from the parameters which can be used to elicit perceptions represented by the adjectives in Table 4

ing smoother perceptual experiences would imply using sinusoidal waveforms with lower amplitudes and rough-like textures would be rendered using rectangular waveforms with higher amplitudes. Spatial period could imply finer to coarser rough-like textures, but this relationship remains uncertain for smoothlike textures as there were fewer sinusoidal textures. While this study was conducted on a tablet with ultrasonic friction reduction, we believe the same effects could be obtained with other tactile rendering technologies which allow for at least creating synthetic textures with two waveforms (square and sine) and controllable spatial period.

\section{Conclusion}

Using models designed to investigate the perceptual space of real textures, this study shows that it is possible to investigate simulated textures using the same strategies. We showed that a continuum of smooth/roughness does exist in a given sample of ultrasonic rendered textures, with different degrees of roughness and smoothness. Influence of waveform and amplitude were found to play an important role in perceiving a texture as smooth or rough, and spatial period as a possible modulator of different degrees of roughness or smoothness. For designers, these results are offering insight on how to tune specific parameters such as wave grating, amplitude and period to elicit perceptions of smooth/roughness, but also possible various degrees of roughness, without the need to go through tribological extractions. In future work we will explore other waveforms such as sawtooth or triangle, with a broader range of amplitudes and periods. Finally the association of visual and tactile components in the case of synthetic textures still needs to be addressed.

\section{REFERENCES}

Biet, M., Giraud, F., and Lemaire-Semail, B. (2007). Squeeze film effect for the design of an ultrasonic tactile plate. IEEE transactions on ultrasonics, Ferroelectrics and Frequency control, 54(12):2678-2688.

Brewer, D. J., Meyer, D. J., Peshkin, M. A., and Colgate, J. E. (2016). Viscous textures: Velocity dependence in fingertip-surface scanning interaction. In 2016 IEEE Haptics Symposium (HAPTICS), pages 265-270. IEEE.

Cingel, D. and Piper, A. M. (2017). How parents engage children in tablet-based reading experiences: An exploration of haptic feedback. In Proceedings of the 2017 ACM Conference on Computer Supported Cooperative Work and Social Computing, pages 505-510. ACM.

Culbertson, H., Schorr, S. B., and Okamura, A. M. (2018). Haptics: The present and future of artificial touch sensation. Annual Review of Control, Robotics, and Autonomous Systems, 1:385-409.

Dargahi, J. and Najarian, S. (2004). Human tactile perception as a standard for artificial tactile sensing-a review. The International Journal of Medical Robotics and Computer Assisted Surgery, 1(1):23-35.

Friesen, R. F., Klatzky, R. L., Peshkin, M. A., and Colgate, J. E. (2018). Single pitch perception of multifrequency textures. In 2018 IEEE Haptics Symposium (HAPTICS), pages 290-295. IEEE.

Guest, S., Dessirier, J. M., Mehrabyan, A., McGlone, F., Essick, G., Gescheider, G., Fontana, A., Xiong, R., Ackerley, R., and Blot, K. (2011). The development and 
validation of sensory and emotional scales of touch perception. Attention, Perception, \& Psychophysics, 73(2):531-550.

Holliins, M., Faldowski, R., Rao, S., and Young, F. (1993). Perceptual dimensions of tactile surface texture: A multidimensional scaling analysis. Perception \& psychophysics, 54(6):697-705.

Hollins, M., Bensmaïa, S., Karlof, K., and Young, F. (2000). Individual differences in perceptual space for tactile textures: Evidence from multidimensional scaling. Perception \& Psychophysics, 62(8):1534-1544.

Kalantari, F., Grisoni, L., Giraud, F., and Rekik, Y. (2016). Finding the minimum perceivable size of a tactile element on an ultrasonic based haptic tablet. In Proceedings of the 2016 ACM on Interactive Surfaces and Spaces, pages 379-384. ACM.

Kalantari, F., Gueorguiev, D., Lank, E., Bremard, N., and Grisoni, L. (2018a). Exploring fingers' limitation of texture density perception on ultrasonic haptic displays. In International Conference on Human Haptic Sensing and Touch Enabled Computer Applications, pages 354-365. Springer.

Kalantari, F., Lank, E., Rekik, Y., Grisoni, L., Giraud, F., Rateau, H., Rekik, Y., Lank, E., Grisoni, L., Vezzoli, E., et al. (2018b). Determining the haptic feedback position for optimizing the targeting performance on ultrasonic tactile displays. In IEEE Haptics Symposium (HAPTICS 2018), San Fransisco, United States.

Levesque, V., Oram, L., MacLean, K., Cockburn, A., Marchuk, N. D., Johnson, D., Colgate, J. E., and Peshkin, M. A. (2011). Enhancing physicality in touch interaction with programmable friction. In Proceedings of the SIGCHI Conference on Human Factors in Computing Systems, pages 2481-2490. ACM.

Messaoud, W. B., Bueno, M.-A., and Lemaire-Semail, B. (2016). Textile fabrics texture: from multi-level feature extraction to tactile simulation. In International Conference on Human Haptic Sensing and Touch Enabled Computer Applications, pages 294303. Springer.

Mullenbach, J., Shultz, C., Colgate, J. E., and Piper, A. M. (2014). Exploring affective communication through variable-friction surface haptics. In Proceedings of the SIGCHI Conference on Human Factors in Computing Systems, pages 3963-3972. ACM.

Okamoto, Shogo, H. N. and Ho., H.-N. (2016). Psychophysical dimensions of material perception and methods to specify textural space. In Pervasive Haptics, pages 3-20. Springer.

Okamoto, S., Nagano, H., and Yamada, Y. (2013). Psychophysical dimensions of tactile perception of textures. IEEE Transactions on Haptics, 6(1):81-93.

Picard, D., Dacremont, C., Valentin, D., and Giboreau, A. (2003). Perceptual dimensions of tactile textures. Acta psychologica, 114(2):165-184.

Rao, A. R. and Lohse, G. L. (1996). Towards a texture naming system: Identifying relevant dimensions of texture. Vision Research, 36(11):1649-1669.

Samur, E., Colgate, J. E., and Peshkin, M. A. (2009). Psychophysical evaluation of a variable friction tactile in- terface. In Human vision and electronic imaging XIV, volume 7240 , page $72400 J$. International Society for Optics and Photonics.

Tiest, W. M. B. and Kappers, A. M. (2006). Analysis of haptic perception of materials by multidimensional scaling and physical measurements of roughness and compressibility. Acta psychologica, 121(1):1-20.

Vezzoli, E., Vidrih, Z., Giamundo, V., Lemaire-Semail, B., Giraud, F., Rodic, T., Peric, D., and Adams, M. (2017). Friction reduction through ultrasonic vibration part 1: Modelling intermittent contact. IEEE transactions on haptics, 10(2):196-207.

Wilson, G. and Brewster, S. A. (2017). Multi-moji: Combining thermal, vibrotactile \& visual stimuli to expand the affective range of feedback. In Proceedings of the 2017 CHI Conference on Human Factors in Computing Systems, pages 1743-1755. ACM. 